\section{Spatio-Temporal Interpolation of UAV Sensor Data}

Gl_Forum 2017, Issue 1

Page: 141 - 156

Full Paper

Corresponding Author:

dariia.strelnikova@edu.fh-kaernten.ac.at

DOI: 10.1553/giscience2017_01_s141

\author{
Dariia Strelnikova and Karl-Heinrich Anders \\ Department of Geoinformation and Environmental \\ Carinthia University of Applied Sciences, Villach, Austria
}

\begin{abstract}
The nature of continuous fields implies the necessity to study them through sampling. Further, spatial interpolation allows the estimation of values for points that were originally discarded. Purely spatial interpolation disregards the temporal component of captured data. Such an approach, however, may lead to wrong conclusions if the data being studied is change-prone. This paper analyses spatio-temporal interpolation of air temperature data captured by an unmanned aerial vehicle (UAV). Since each point in the resulting dataset has a unique timestamp, purely spatial interpolation of UAV sensor data is likely to be less accurate than spatio-temporal interpolation. In the case of temperature data, for instance, spatio-temporal interpolation makes it possible to monitor the movement of pockets of warm air. Geoinformation systems, like ArcGIS or QGIS, have rich spatial interpolation toolsets. These systems, however, provide no native spatio-temporal interpolation means. We explored spatio-temporal interpolation of UAV data and developed a prototype of a stand-alone interpolation tool that exploits radial basis functions (RBF) and inverse distance weighting (IDW) for interpolation in a continuous space-time domain. This paper discusses spatio-temporal interpolation of UAV data in comparison with purely spatial interpolation, as well as the application of the developed tool.
\end{abstract}

\title{
Keywords:
}

spatio-temporal interpolation, UAV, sensor data, radial basis functions, IDW.

\section{Introduction}

Most phenomena on Earth, like air temperature or humidity, change over time. Temporal attributes of geodata are often crucial for the understanding of this data and its proper application. As our world is infinitely complex, it is rarely possible to study its phenomena based on comprehensive data (Longley et al., 2010). When a phenomenon of interest is a continuous field, sampling is the only way to study it. Spatial interpolation methods enable the estimation of values in unsampled points and the description of a phenomenon as a whole. 
It is important to differentiate between a purely spatial interpolation and spatio-temporal interpolation (STI). The first is aimed at calculating a value of interest by processing the corresponding values of the nearby points in space, disregarding the temporal component of the data under study. The second approach additionally takes into consideration the remoteness of the measurement points in time. Interpolating spatial and temporal aspects of data separately, one aspect at a time, is, according to Pebesma (2012) and Li et al. (2016), a popular approach to the STI problem. A possible reason is the lack of software tools that offer interpolation in a continuous space-time domain.

The goal of this study was to explore possible benefits of STI in comparison with purely spatial interpolation when applied to UAV sensor data. To achieve this, we developed a prototype of an easy-to-use tool with STI functionality (STI-Tool) and assessed its performance on air temperature data captured by a UAV. Tenfold cross-validation together with the visualization of interpolation results served for quality assessment.

This paper is structured as follows. Section 2, 'STI in the Literature and STI-Software', presents the theoretical background of STI and information on available STI implementations. In Section 3, 'Choice of STI Approach and Methods', we explain the methodology behind the STI-Tool. 'Implementation of the STI-Tool' describes the development of the prototype. Section 5, 'Comparison of STI with Purely Spatial Interpolation and Quality Assessment', presents the results of comparing various interpolation methods and discusses the quality assessment of the results. Finally, in 'Conclusions and Perspectives', we sum up the study and suggest directions for its further development.

\section{STI in the Literature and STI-Software}

While spatial interpolation has been thoroughly studied, STI has been much less explored. Most of STI studies have taken place in the last 20 years. In 'Interpolation Methods for Spatio-Temporal Geographic Data', Li and Revesz (2004) stated that there was only one published study on spatio-temporal interpolation they could find: the work of Eric J. Miller, who developed and implemented four-dimensional geostatistical kriging (Miller, 1997). Spatio-temporal interpolation was then studied further. Hussain et al. (2010) offered a BoxCox transformed hierarchical Bayesian multivariate spatio-temporal interpolation method predicting precipitation in Pakistan. Srinivasan et al. (2010) achieved substantial improvements in computational performance of spatio-temporal kriging. Pebesma (2012) presented spatio-temporal classes of R package gstat that could be used for STI. Lguensat et al. (2014) developed an STI model for sea surface temperature. Li et al. (2014) implemented two spatio-temporal inverse distance weighting (IDW) based methods in order to assess the trends of fine particulate matter concentration, and discussed ways to achieve computational efficiency while dealing with large datasets. Montero-Lorenzo et al. (2013) also studied air pollution and showed that spatio-temporal strategies lead to more accurate predictions than purely spatial or purely temporal strategies. Graeler et al. (2016) developed an extension to an $\mathrm{R}$ package gstat with spatio-temporal covariance functions for spatio-temporal kriging. The topic of STI is attracting more attention. Most of the studies, however, consider only geodata 
referenced to a 2-dimensional frame, and it has also been pointed out that '[a] spatiotemporal model is not guaranteed to outperform purely spatial predictions' (Graeler et al., 2016, p. 216).

Specialists often treat spatio-temporal interpolation as a sequence of snapshots of spatial interpolations' (Li et al., 2014, p. 9103). Although in some cases STI with space and time treated independently can yield good results (Susanto et al., 2016), many scientists (Ferreira et al., 2000; Li et al., 2014; Montero-Lorenzo et al., 2013) expect that conducting STI in a continuous space-time domain may result in higher interpolation quality.

Geoinformation systems (GIS) often include a rich set of tools for spatial interpolation, e.g. Geostatistical Analyst in ArcGIS (Krivoruchko, 2011), but no or few native STI tools (Li et al., 2016). Classes and methods for spatio-temporal kriging written in $\mathrm{R}$ language (Graeler et al., 2016; Pebesma, 2004) expand the STI functionality of GIS. They are available via RBridge in ArcGIS (ESRI, 2016) and as command line tools in GRASS GIS. In both cases, work with the $\mathrm{R}$ tools requires users to have programming skills and knowledge of the $\mathrm{R}$ language. Thus many GIS users may experience difficulties with this approach. To provide more users with STI means, an STI tool must be intuitive and have a simple graphical user interface (GUI). For this reason, while working on the application of STI to UAV sensor data, we also aimed to create a user-friendly tool.

The literature reveals over 40 spatial interpolation methods (Li \& Heap, 2008), including IDW (Isaaks \& Srivastava, 1989; Li et al., 2014), shape functions (Li et al., 2016; Li \& Revesz, 2004), radial basis functions (Hickernell \& Hon, 1999; Škala, 2016), splines (de_Boor, 2001; Mitas \& Mitasova, 1999), local trend surfaces (Akima, 1978; Cleveland \& Devlin, 1988; Venables \& Ripley, 2002), natural neighbours (Sibson, 1981) and kriging (Cressie, 1990; Oliver \& Webster, 1990; Srinivasan et al., 2010; Stein, 2013). The following section explains the choice of interpolation methods used in this study. It also explains the approach to the transformation of a spatio-temporal interpolation problem into a 4D spatial interpolation problem.

\section{Choice of STI Approach and Methods}

In STI there are two approaches to the temporal component of data. The so-called extension approach, proposed by Li (2003), treats time as another dimension in space. Another approach, called a reduction approach, treats time independently (Susanto et al., 2016). The logic behind the STI-Tool rests on the extension approach, for the following reasons:

1. It models reality more accurately, as every measurement in the real world is done in both time and space simultaneously.

2. It reflects the nature of the UAV sensor data, where each location has a different timestamp. Interpolation first in space and then in time requires either (1) interpolating all target point values from a single point or (2) treating subsets of sampled locations as existing in time-space simultaneously, disregarding their temporal attributes, which contradicts the very concept of STI. 
Susanto et al. (2016) provide detailed information on interpolation methods used in environmental modelling. Their analysis shows that kriging has been reported as the most suitable spatial interpolation method in many studies. The downside of kriging is its computational complexity. According to Susanto et al. (2016), Curtarelli et al. (2015) and Li et al. (2014), IDW is comparable with kriging, outperforming it in some cases, and its computational efficiency is much higher. IDW can be used for $4 \mathrm{D}$ data, and was therefore the first choice for this study. As IDW produces local extrema at known locations (Mitas \& Mitasova, 1999), we considered it reasonable to use one more interpolation method for comparison.

Splines were presented by Mitasova et al. (1995) as a good method of interpolation and approximation of $\mathrm{d}$-dimensional scattered point data to $\mathrm{d}$-dimensional grids. Although splines can produce peaks, waves and pits, they are considered 'rather successful' (Mitas \& Mitasova, 1999, p. 487) for climate data interpolation, as those peaks and pits can be smoothened. Smoothness is obtained by a variety of approaches, e.g. a variational approach (Mitas \& Mitasova, 1999) or radial basis functions (RBFs) (Škala, 2016) that 'can be interpreted as roughness-minimizing splines' (Hickernell \& Hon, 1999, p. 1). As RBFs are 'one of the primary tools for interpolating multidimensional scattered data' (Wright 2003, p. iii), we use interpolation by means of RBF along with IDW.

\section{Implementation of the STI-Tool}

\section{Data}

The UAV sensor data used in this study results from a survey near Noetsch im Gailtal in the south of Austria. Each data entry contains latitude and longitude (in decimal degrees), altitude (in metres), timestamp (date and time), and three measurement values: air temperature, atmospheric pressure and humidity. The interpolation results presented in this paper are based on air temperature values.

Figure 1 shows the correlation between temperature values (expressed as colour), altitude ( $\mathrm{Y}$ axis) and measurement time (X-axis). Air temperatures captured in the first half of the flight differ from temperatures captured at the same altitudes during the second half of the flight. Thus purely spatial interpolation is likely to be less accurate than STI. 


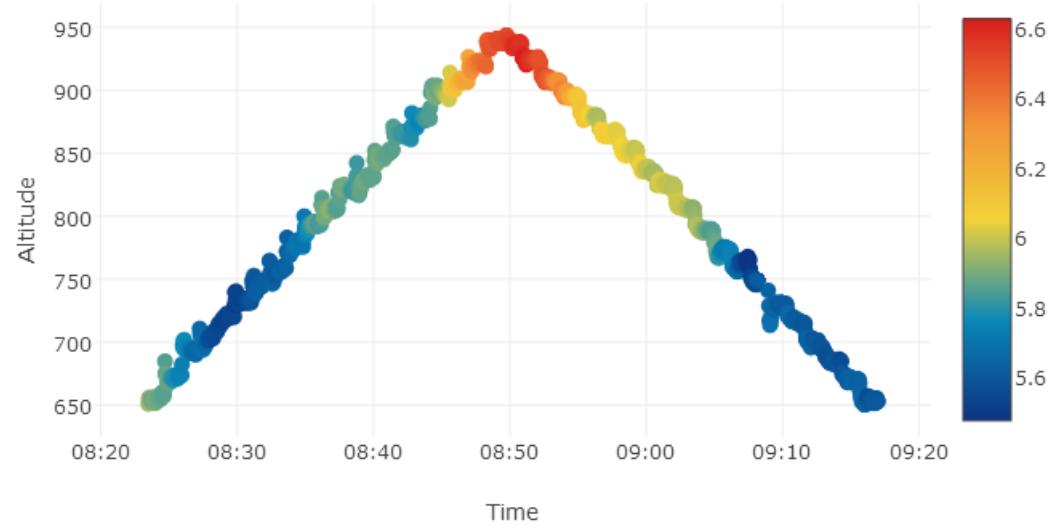

Figure 1: Correlation Between Temperature Values, Altitude and Measurement Time

Figure 2 displays the flight trajectory. The temperature values corresponding to the sampled locations are expressed in colour.

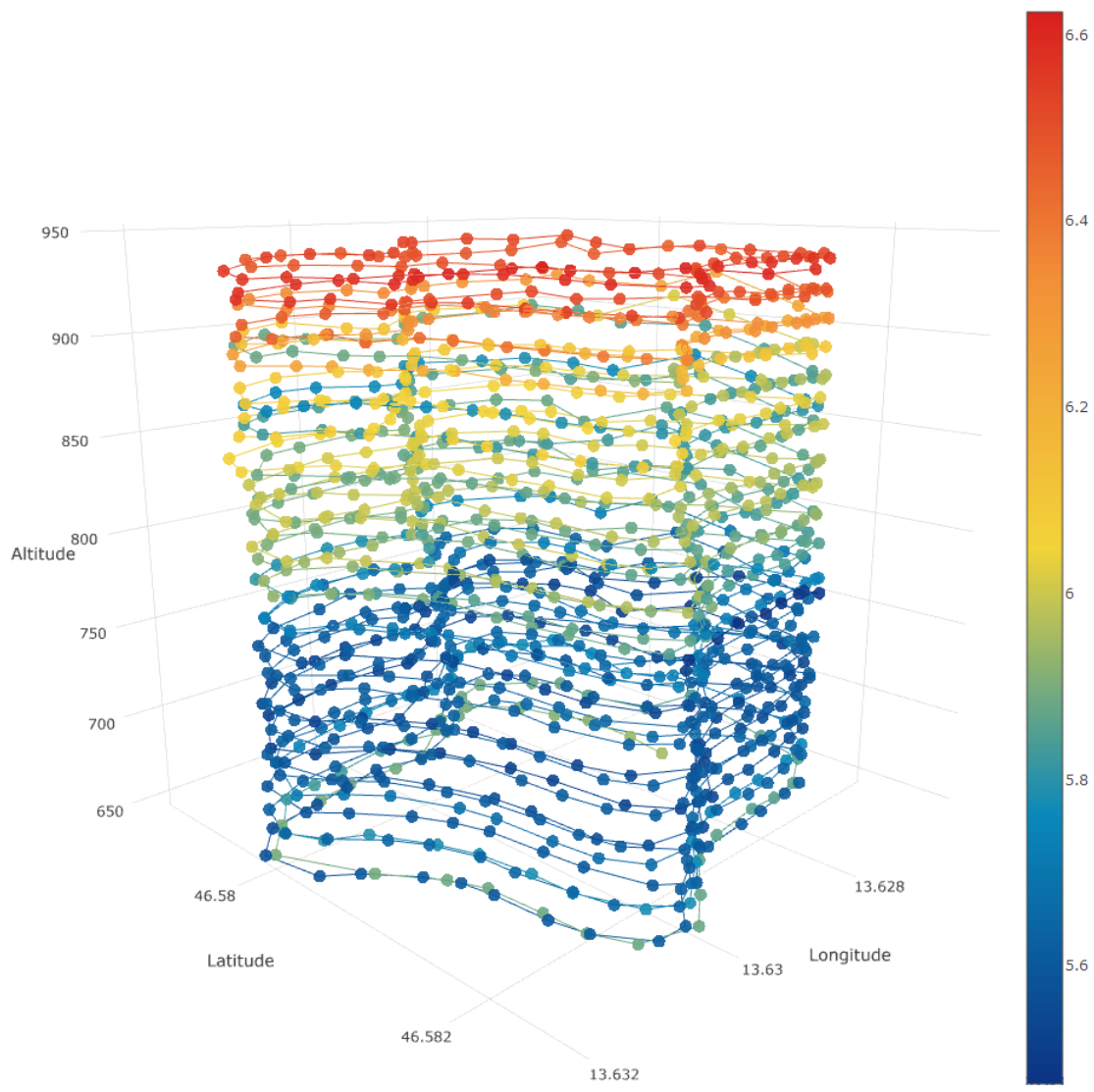

Figure 2: Air Temperature Values Along the Flight Trajectory 
The UAV sensor data has the following important characteristics:

- It is irregular both spatially and temporally.

- Most of the locations in our dataset are sampled just once.

- Locations are referenced to a three-dimensional spatial frame.

\section{Normalization}

As transformation of geographic coordinates into projected coordinates was outside the scope of the STI-Tool functionality, we used normalization to unify distance units. This also simplified the visualization of interpolated data. Normalization preserves topology. Minimum and maximum values of corresponding coordinates are provided in the form of metadata in the output JSON file. This allows the restoration of the original shape of the study area if desired. Normalization exploits the following formula:

$$
\text { normalized value }_{i}=\frac{\text { observed value }_{i}-\text { minimum observed value }}{\text { maximum observed value }- \text { minimum observed value }}
$$

After normalization, all the coordinate values belong to the interval $[0 ; 1]$. Edges of the bounding hexahedron of the input data points have length 1 (one). Last, we normalized timestamps by analogy with normalization of coordinates.

\section{Transformation of the Interpolation Problem into 4D}

The extension approach (Li et al., 2016) is based on the transformation of time units into space units. It involves multiplication of timestamps by an empirically chosen factor C (time scale) measured in $\frac{\text { distance units }}{\text { time units }}$. Conversion of timestamps in date-time format into Unix time (Hayes, 2015) enables the necessary calculations. The extension approach is similar to Minkowski's spacetime concept (Minkowski, 1909, trans. 2012). Li et al. (2016) carried out their analysis using four time scales: $1,1 / 10,1 / 5$ and $1 / 15$. They chose $C=1 / 10$ as it resulted in the best values for error statistics. As they note, the $C$ factor has to be chosen experimentally for each particular dataset.

\section{Technical Solution}

The STI-Tool was developed in Python with the use of scipy libraries (https://www.scipy.org/). The visualization of interpolation results given in this paper involved the use of plotly (https://plot.ly/python/). Utilization of the $\mathrm{k}$-d tree data structure, introduced by Bentley (1975) and known for solving the nearest-neighbour problem in logarithmic time (Samet, 1995), enabled quick nearest-neighbour lookup. The Python implementation of k-d tree is the class KDTree in the package scipy.spatial. This class uses an algorithm by Maneewongvatana and Mount (1999). The Python RBF implementation is the Rbf class in the scipy.interpolate package. This class carries out the interpolation using the algorithms of Hetland and Travers (2006-2007). The STI-Tool 
exploits this class for RBF interpolation and includes its own IDW implementation. The use of open source libraries enables the reproduction of analyses conducted with the STI-Tool in a Python console.

\section{Accuracy Assessment}

The STI-Tool employs the following statistics to assess interpolation quality: (1) Mean Absolute Error (MAE), (2) Mean Squared Error (MSE), (3) Root Mean Squared Error (RMSE), (4) Mean Absolute Relative Error (MARE), and (5) Coefficient of Determination (R2). These statistics are often used for interpolation error assessment (Curtarelli et al., 2015; Graeler et al., 2016; Keller et al., 2015; Li \& Heap, 2008; Li \& Revesz, 2002; Susanto et al., 2016; Tomczak, 1998; Wise, 2011), with RMSE being the most popular. The calculation formulas were adopted from Li et al. (2016) and Keller et al. (2015). The STI-Tool assesses the accuracy of the chosen interpolation methods through a tenfold cross-validation approach, which, according to Efron and Tibshirani (1997), provides accurate information on the size of interpolation error.

\section{Comparison of STI with Purely Spatial Interpolation and Quality Assessment}

The study involved testing four interpolation methods: spatio-temporal or purely spatial IDW, spatio-temporal or purely spatial RBF. The STI-Tool gives access to these interpolation methods by means of a simple GUI (Figure 5). The availability of spatial interpolation methods provided means to compare spatial and spatio-temporal interpolation results.

Our input data was in CSV format. The STI-Tool automatically fills in the names of columns representing parameters used for interpolation in GUI dropdown lists (e.g. column with the name 'lat' for latitude) after the user has chosen an input file. Users can adjust the suggested parameters. The results of interpolation are saved to a JSON file. It is also possible to convert input CSV data into JSON format, e.g. in order to enable simultaneous visualization. 


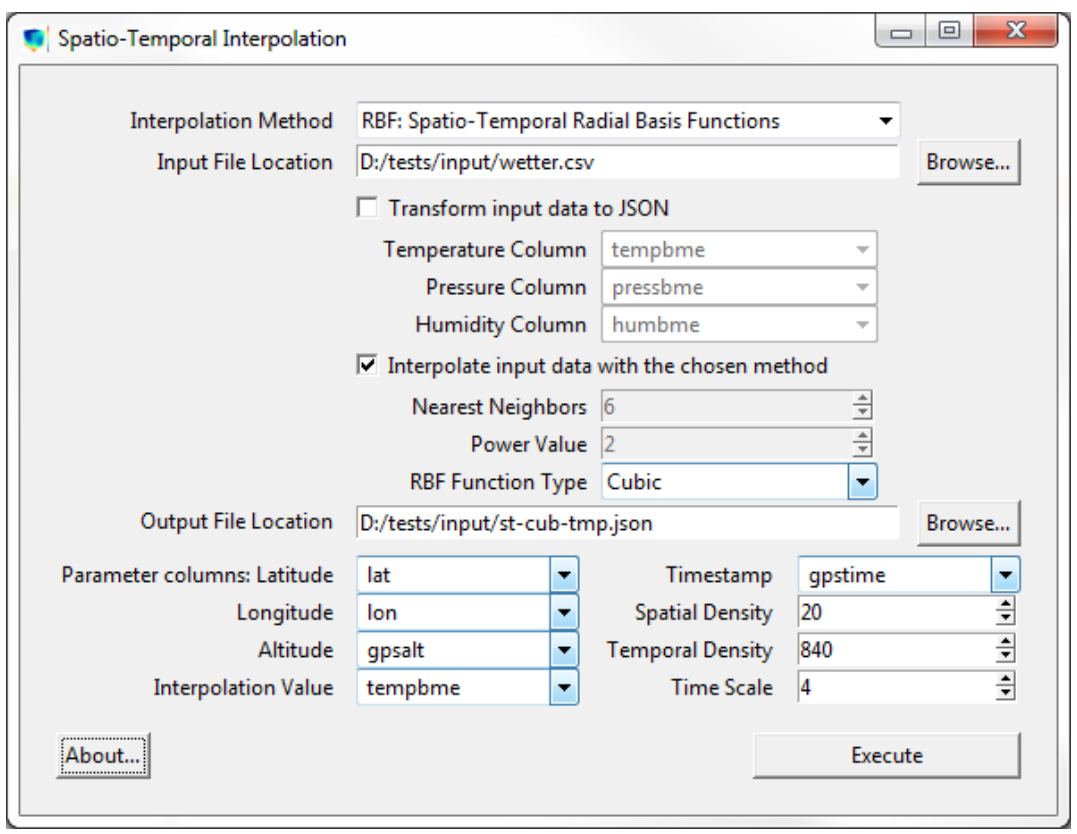

Figure 3: STI-Tool Graphical User Interface

IDW interpolation results depend on the number of nearest neighbours $(\mathrm{N})$ and power $(\mathrm{P})$. During the tool development, we tested different values for these parameters: number of nearest neighbours from 2 to 100, and power from 1 to 10. Comparison of error statistics revealed that the best combinations of parameter values are different for spatial interpolation and STI, and also depend on the dataset used for interpolation. High power values in our tests are associated with less accurate interpolation results.

Along with IDW, we tested seven radial basis functions within the RBF interpolation method: three piecewise smooth (thin plate, linear, cubic) and four infinitely smooth (inverse, quantic, multiquadric and Gaussian). Error statistics for IDW, thin plate and linear RBFs have low values for both spatio-temporal and spatial interpolation. Other RBFs conduct purely spatial interpolation with large errors. At the same time, cubic function has the best STI error statistics.

Table 1: Comparison of Error Statistics for Spatial Interpolation and STI

\begin{tabular}{|c|c|c|c|c|c|c|c|c|c|c|}
\hline \multirow[t]{2}{*}{ Function } & \multicolumn{5}{|c|}{ Spatial Interpolation } & \multicolumn{5}{|c|}{ Spatio-Temporal Interpolation $(C=1)$} \\
\hline & MAE & MSE & RMSE & MARE & $k^{2}$ & MAE & MSE & RMSE & MARE & $\mathrm{R}^{2}$ \\
\hline $\mathrm{DW}(\mathrm{N}=6, \mathrm{P}=1)$ & 0.1378 & .0673 & 0.2595 & 0.0219 & 0.9076 & 0.0535 & 0.0158 & 0.1256 & 0.0082 & 0.9779 \\
\hline ubic RBF & .5955 & 6.2740 & 5.8025 & 0.0840 & 0.1518 & 0.0268 & 0.0043 & & & 0.9946 \\
\hline inear RBF & 1428 & .0603 & 0.2457 & 0.0231 & 0.9084 & 0.0434 & 0.0119 & 0.1092 & 0.0067 & 0.9830 \\
\hline hin plate RBF & 0.1573 & .1304 & 0.3611 & 0.0251 & 0.8215 & 0.0319 & 0.0064 & 0.0802 & 0.0050 & 0.9912 \\
\hline Ruintic RBF & 40.311 & $9 \times 10^{4}$ & $3 \times 10^{2}$ & 6.2236 & 0 & 0.1181 & 0.3272 & 0.5720 & 0.0176 & 0.7245 \\
\hline hultiquadric RBF & $6 \times 10^{2}$ & $3 \times 10^{7}$ & $5.5 \times 10^{3}$ & 91.650 & 0 & 28.0252 & $9 \times 10^{4}$ & $3 \times 10^{2}$ & 3.6612 & 0.0000 \\
\hline Baussian RBF & $6 \times 10^{3}$ & $4 \times 10^{9}$ & $7 \times 10^{4}$ & $9 \times 10^{2}$ & 0 & $1 \times 10^{2}$ & $2 \times 10^{6}$ & $1 \times 10^{3}$ & 13.327 & 0.0000 \\
\hline hverse RBF & $5 \times 10^{3}$ & $1 \times 10^{10}$ & $1 \times 10^{5}$ & $6 \times 10^{2}$ & 0 & 1.1541 & $1.5 \times 10^{2}$ & 12.124 & 0.1538 & 0.0539 \\
\hline
\end{tabular}


Table 1 displays error statistics for seven RBFs and IDW with parameters $\mathrm{N}=6, \mathrm{P}=1$. These IDW parameters yield the best spatial interpolation results and second best (after $\mathrm{N}=4, \mathrm{P}=1$ ) STI results. Errors of STI are lower in comparison with spatial interpolation, which indicates that STI has higher interpolation accuracy. Error values vary with the chosen time scale. Table 1 gives STI results for $C=1$. For our dataset, these results are improved if $C=4$ (Table 2).

Table 2: Error Statistics for Time Scale C $=4$

\begin{tabular}{|l|l|l|l|l|l|}
\hline Function & MAE & MSE & RMSE & MARE & $R^{2}$ \\
\hline Cubic RBF & 0.0086 & 0.0002 & 0.0150 & 0.0014 & 0.9997 \\
\hline Thin plate RBF & 0.0111 & 0.0005 & 0.0227 & 0.0018 & 0.9992 \\
\hline Linear RBF & 0.0204 & 0.0031 & 0.0526 & 0.0031 & 0.9954 \\
\hline Quintic RBF & 0.0210 & 0.0041 & 0.0585 & 0.0033 & 0.9938 \\
\hline IDW(N=4. $\mathrm{P}=1)$ & 0.0300 & 0.0067 & 0.0800 & 0.0046 & 0.9901 \\
\hline IDW(N=6. $\mathrm{P}=1)$ & 0.0327 & 0.0079 & 0.0874 & 0.0049 & 0.9879 \\
\hline
\end{tabular}

The STI-Tool enables calculation of error statistics from GUI upon completion of the interpolation (Figure 4). It evaluates errors based on the interpolation method used and configuration of parameters. The tool presents the results in the form of a message dialog. In addition to the statistics described above, it calculates and displays the percentage of interpolated values that lie within the initial value range.

Interpolated

File wetter_uav.csv was successfully interpolated and saved under :

D:/tests/input/st-cub-tmp.json

Number of non-duplicate observations: 1377

Latitude: from 46.578825 to 46.582613333

Longitude: from 13.626273333 to 13.632271667

Altitude: from 613.6 to 943.9

Start: 11.11.2016 08:23:29

End: 11.11.2016 09:20:04

Temporal step: 840 seconds

Spatial density: $20 \times 20 \times 20$

Phenomenon: Air Temperature

Input values: from 5.47116349981 to 6.62240574642

Interpolated values: from 4.86355589728 to 5.96212117343

Do you want to calculate interpolation error?

Figure 4: Message Generated on Completion of Interpolation 
Error statistics given by Tables 1 and 2 imply high interpolation quality. As seen in Figure 4, some of the interpolated values are significantly below the initial minimum, and there are no interpolants reaching the input maximum. It is expected that RBF interpolation may result in values outside of the initial range. These values, however, are not expected to vary substantially from the original observations. Thus we can surmise that a standard error assessment approach does not comprehensively indicate interpolation accuracy in cases of STI of UAV sensor data. The visualization of results of the linear RBF STI supports this assumption (Figure 5).

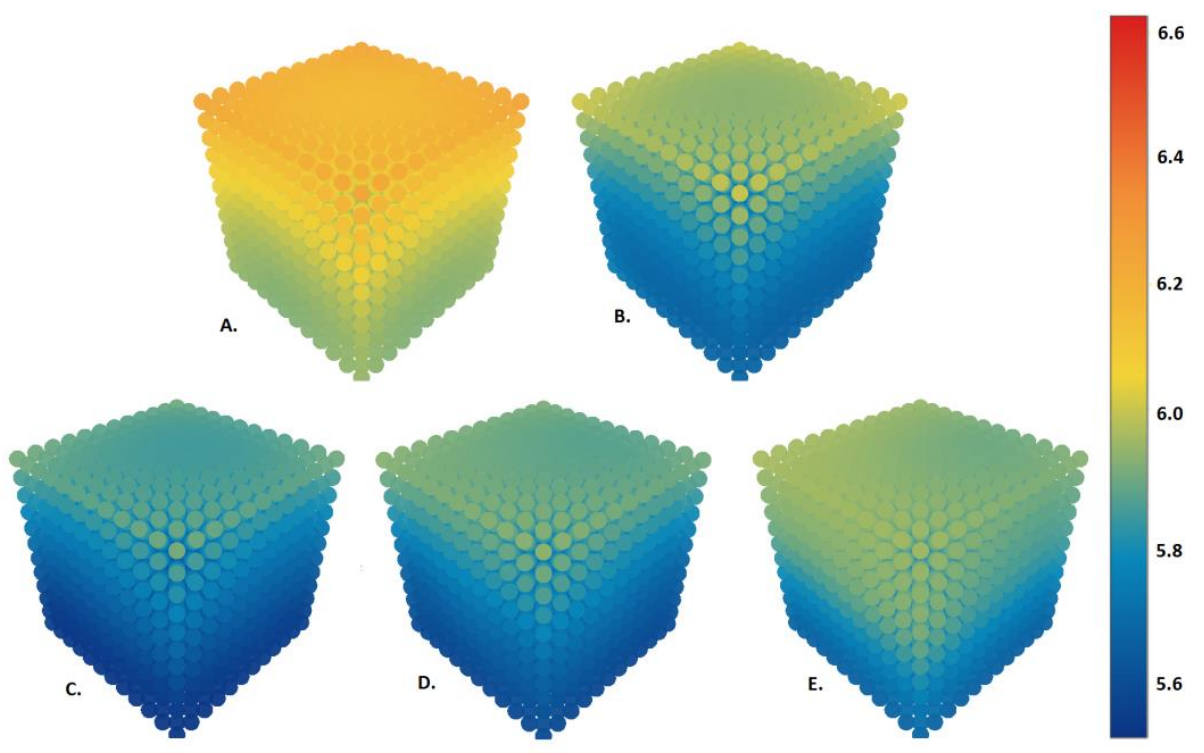

Figure 5: Visualization of Linear RBF STI Results, C = 4

The interpolated cube corresponding to the beginning of the flight (Figure 5A) has many overestimated temperature values. In the middle of the flight (Figure $5 \mathrm{C}$ ), values tend to be underestimated. These RBF interpolation results are inconclusive, which can be attributed to the irregularity and sparseness of the input data. As only the outer faces of the cubes are visible, it is impossible to visually assess the interpolation quality of inner points. 


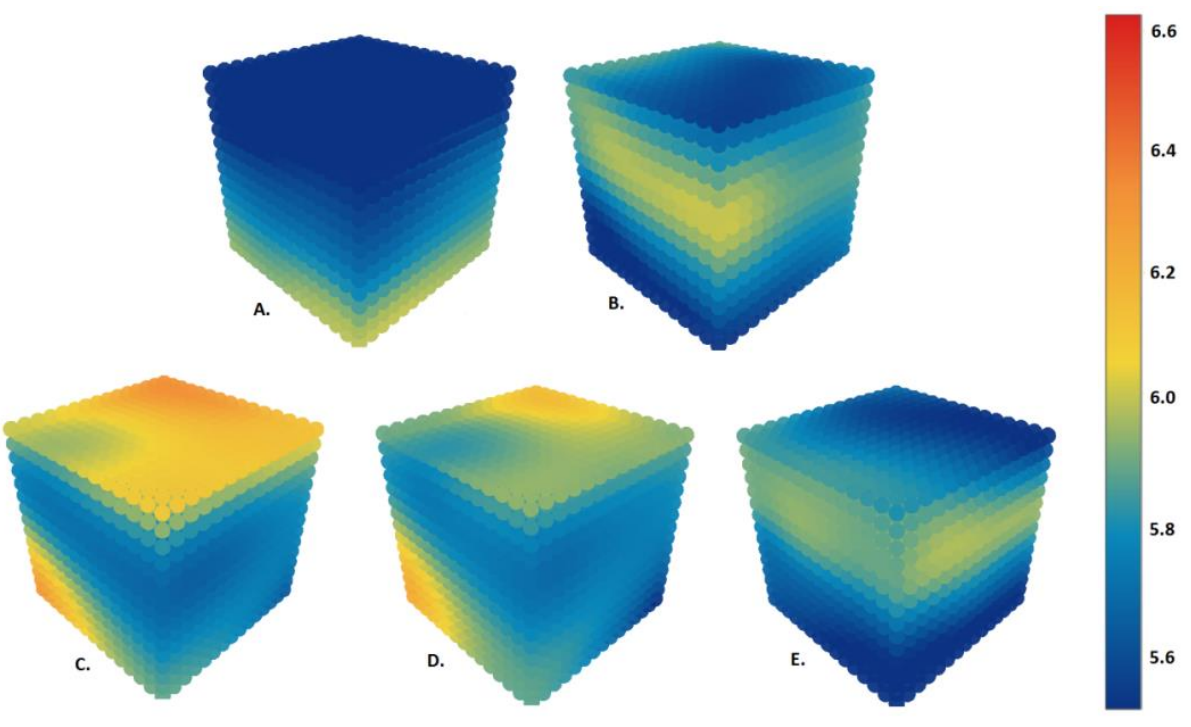

Figure 6: Visualization of Cubic RBF STI Results, C = 4

Figure 6 displays the results of spatio-temporal cubic RBF interpolation. Interpolation results can be visually interpreted as plausible, displaying a moving pocket of warm air that eventually cools down. Since the air is not expected to form even layers of equal temperature, unambiguous visual assessment of interpolation quality is difficult.

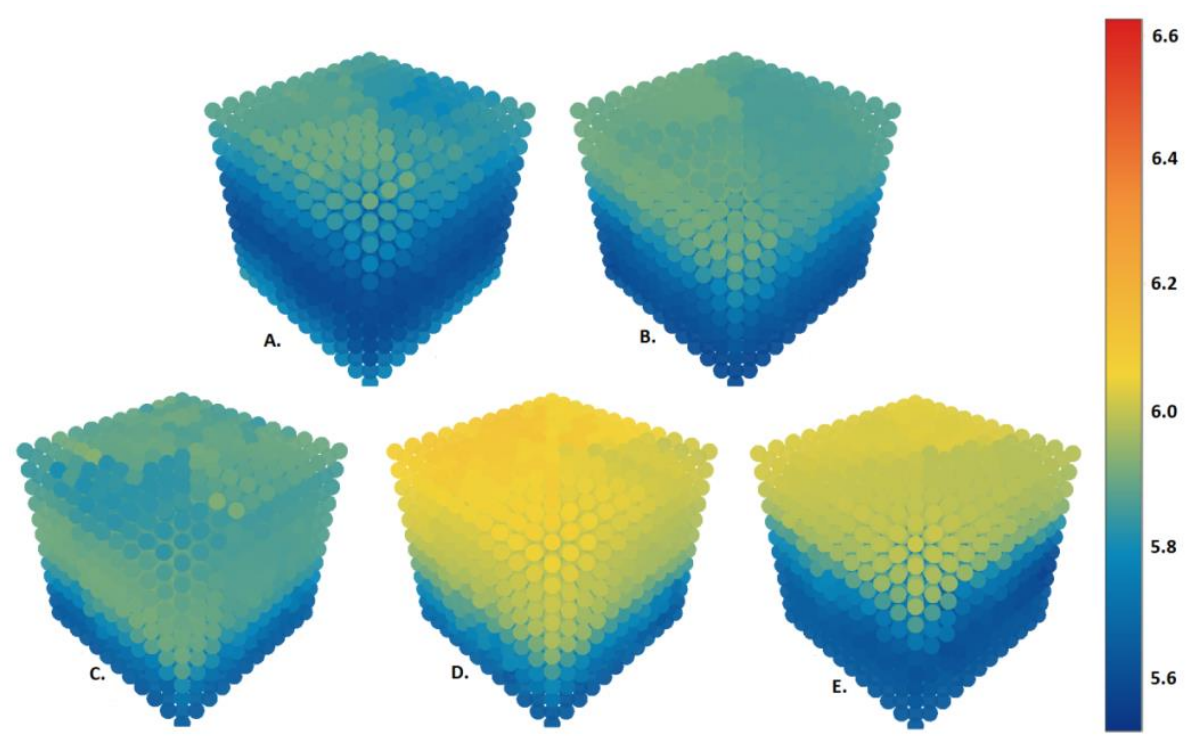

Figure 7: Spatio-Temporal Interpolation with IDW, C = 4 
Figure 7 visualizes the results of STI by means of IDW. Interpolated bounding cube D contains many overestimated values. The results of purely spatial IDW are given by Figure 8 .

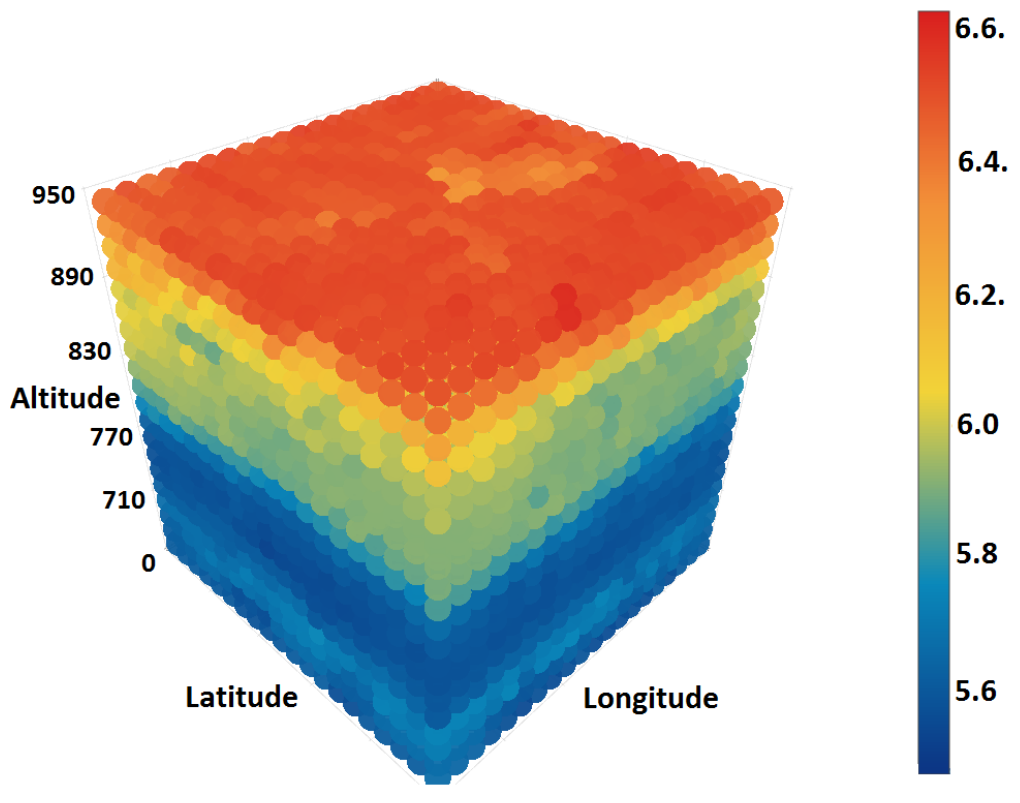

Figure 8: Visualization of results of purely spatial IDW $(N=6, P=1)$

Figure 8 indicates that temperature values for altitudes from 800 to 900 metres are averaged. An increase in temperature during the second half of the flight cannot be identified when the temporal component of data is discarded. An even more substantial distortion takes place when time is disregarded during interpolation with RBF (Figure 9):

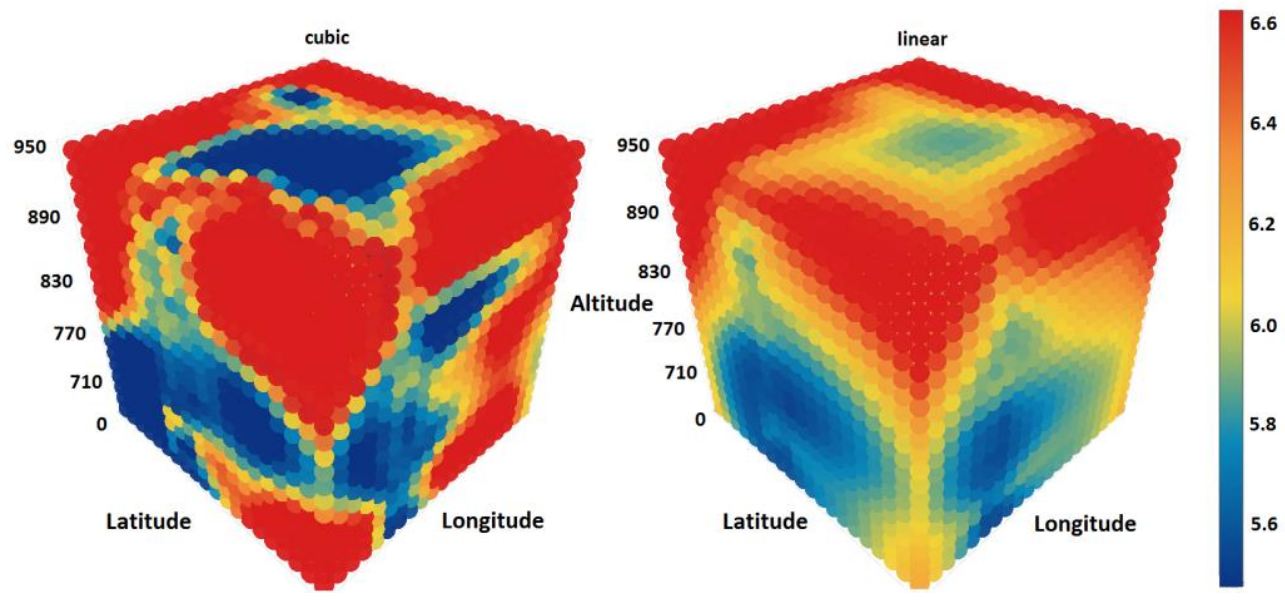

Figure 9: Visualization of results of purely spatial cubic and linear RBF 
Large volumes in the interpolated bounding cube that are located outside the flight trajectory have overestimated values. The interpolation results can be visually interpreted as inaccurate. Therefore, we can see that if a phenomenon under study experiences change within a measurement interval, discarding the temporal attribute during interpolation can lead to inaccurate and invalid results. This is less obvious when IDW interpolation is used but becomes evident when data is interpolated using RBF.

\section{Conclusion and Perspectives}

Within this study, we performed spatial and spatio-temporal interpolation of UAV sensor data by means of the spatio-temporal interpolation tool (STI-Tool). The tool provides an easy-to-use GUI for choosing interpolation methods and adjusting interpolation parameters. This paper presents results for the interpolation of air temperature data. The STI-Tool was additionally tested with humidity and air pressure data and is considered to be suitable for other regular and irregular 3-dimensional scattered point data of ratio nature. For the dataset tested, Inverse Distance Weighting performed considerably better than Radial Basis Functions in cases of spatial interpolation, and cubic RBF yielded the most accurate results in the case of STI. The study showed that irrespective of the interpolation method used, spatio-temporal interpolation results in more accurate predictions.

Error statistics calculated under the tenfold cross-validation approach suggest low interpolation errors. The visualization of interpolation results, however, shows that low values for error statistics do not necessarily mean accurate value prediction in every spatiotemporal point of the study area. Thus, possible directions for further study are:

- Raising the STI quality

- Improving validation procedures

- Making the STI-Tool accessible to a wide range of GIS users.

The following steps can be taken to raise spatio-temporal interpolation quality:

1. Shaping the flight trajectory in such a way that a bounding hexahedron requires little extrapolation, or discarding some sample points before the bounding hexahedron calculation

2. Using two UAVs to capture data within one study region simultaneously and in such a way that remotely located points are captured at the same time

3. Capturing vertical atmospheric structure simultaneously with the UAV flight (e.g. by means of a tethered balloon or a Small Unmanned Meteorological Observer) and using it as an input together with trajectory data.

Possible improvements of the validation procedures are:

1. Using points from one UAV trajectory as a training subset, and points of another UAV trajectory or a vertical atmospheric profile as a validation subset

2. Creating synthetic spatio-temporal reference data, sampling it along different trajectories at different times and reconstructing this synthetic data from sampled 
subsets for evaluation purposes. This approach has the advantage of providing means for trajectory adjustments, leading to better interpolation results.

In view of the lack of easy-to-use spatio-temporal interpolation software, the STI-Tool tool can be rega6rded as the first step in the creation of free, easy-to-use means of spatiotemporal interpolation of data referenced to a 3D spatial frame. Its further development may include providing additional options, such as storing used parameters to simplify analysis reproduction, or saving calculated error statistics. It might also be possible to transform the STI-Tool into a QGIS plug-in, thus making it accessible to a wide range of GIS users.

\section{References}

Akima, H. (1978). A method of bivariate interpolation and smooth surface fitting for irregularly distributed data points. ACM Transactions on Mathematical, 4(2), 148-159.

Bentley, J. L. (1975). Multidimensional binary search trees used for associative searching. Communications of the ACM, 18(9), 509-517.

Cleveland, W. S., \& Devlin, S. J. (1988). Locally weighted regression: an approach to regression analysis by local fitting. Journal of the American Statistical Association, 83(403), 596-610.

Cressie, N. (1990). The origins of kriging. Mathematical Geology, 22(3), 239-252. https://doi.org/10.1007/BF00889887

Curtarelli, M., Leão, J., Ogashawara, I., Lorenzzetti, J., \& Stech, J. (2015). Assessment of Spatial Interpolation Methods to Map the Bathymetry of an Amazonian Hydroelectric Reservoir to Aid in Decision Making for Water Management. ISPRS International Journal of Geo-Information, 4(1), 220-235.

de_Boor, C. A. (2001). Practical Guide to Splines (Vol. 27). Berlin/Heidelberg, Germany: Springer.

Efron, B., \& Tibshirani, R. (1997). Improvements on Cross-Validation:: The.632+ Bootstrap Method. Journal of the American Statistical Association, 92(438), 548-560.

ESRI. (2016). R - ArcGIS Community: A community driven collection of free, open source projects making it easier and faster for $\mathrm{R}$ users to work with ArcGIS data, and ArcGIS users to leverage the analysis capabilities of R. Retrieved from https://r-arcgis.github.io/

Ferreira, F., Tente, H., Torres, P., Cardoso, S., \& Palma-Oliveira, J. M. (2000). Environmental Monitoring and Assessment, 65(1/2), 443-450. https://doi.org/10.1023/A:1006433313316

Graeler, B., Pebesma, E., \& Heuvelink, G. (2016). Spatio-Temporal Interpolation using gstat. The R Journal, 8(1), 204-218.

Hayes, D. R. (2015). A Practical Guide to Computer Forensics Investigations. Indianapolis, Indiana, USA: Pearson.

Hetland, R., \& Travers, J. (2006-2007). rbf.py: Radial basis functions for interpolation/smoothing scattered n-dimensional data. Package scipy.interpolate.

Hickernell, F. J., \& Hon, Y. C. (1999). Radial basis function approximations as smoothing splines. Applied Mathematics and Computation, 102(1), 1-24.

Hussain, I., Spöck, G., Pilz, J., \& Yu, H.-L. (2010). Spatio-temporal interpolation of precipitation during monsoon periods in Pakistan. Advances in Water Resources, 33(8), 880-886.

Isaaks, E. H., \& Srivastava, R. M. (1989). Applied Geostatistics. New York: Oxford University.

Keller, J. P., Olives, C., Kim, S.-Y., Sheppard, L., Sampson, P. D., Szpiro, A. A.,\& Kaufman, J. D.

(2015). A unified spatiotemporal modeling approach for predicting concentrations of multiple air pollutants in the multi-ethnic study of atherosclerosis and air pollution. Environmental health perspectives, 123(4), 301-309. 
Krivoruchko, K. (2011). Spatial statistical data analysis for GIS users. Dvdr edition. London: ESRI Press.

Lguensat, R., Tandeo, P., Fablet, R., \& Garello, R. (2014). Spatio-temporal interpolation of Sea Surface Temperature using high resolution remote sensing data. In Oceans 2014 - St. John's (pp. 1-4). [Piscataway, NJ]: IEEE.

Li, J., \& Heap, A. D. (2008). A Review of Spatial Interpolation Methods for Environmental Scientists (Record 2008/23). Canberra, Australia: Geoscience Australia.

Li, L. (2003). Spatiotemporal Interpolation Methods in GIS (Ph.D. Thesis.). Department of Computer Science and Engineering, University of Nebraska, Lincoln.

Li, L., Losser, T., Yorke, C., \& Piltner, R. (2014). Fast inverse distance weighting-based spatiotemporal interpolation: a web-based application of interpolating daily fine particulate matter PM2:5 in the contiguous U.S. using parallel programming and $\mathrm{k}-\mathrm{d}$ tree. International Journal of Environmental Research and Public Health, 11(9), 9101-9141.

Li, L., \& Revesz, P. (2002). A Comparison of Spatio-temporal Interpolation Methods. In Lecture notes in computer science, Lecture notes in artificial intelligence 0302-9743: Vol. 2478.

Geographic information science. Second international conference, GIScience 2002, Boulder, CO, USA, September 25-28, 2002, proceedings / Max J. Egenhofer, David M. Mark (eds.) (pp. 145160). Berlin, London: Springer.

Li, L., \& Revesz, P. (2004). Interpolation methods for spatio-temporal geographic data. Computers, Environment and Urban Systems, 28(3), 201-227.

Li, L., Zhou, X., Kalo, M., \& Piltner, R. (2016). Spatiotemporal Interpolation Methods for the Application of Estimating Population Exposure to Fine Particulate Matter in the Contiguous U.S. and a Real-Time Web Application. International Journal of Environmental Research and Public Health, 13(8), 749-769. Retrieved from http://www.mdpi.com/1660-4601/13/8/749

Longley, P. A., Goodchild, M. F., Maguire, D. J., \& Rhind, D. W. (2010). Geographic information systems and science (3rd ed.). Hoboken, N.J.: Wiley; Chichester : John Wiley [distributor].

Maneewongvatana, S., \& Mount, D. M. (1999). It's Okay to Be Skinny, If Your Friends Are Fat. Center for Geometric Computing 4th Annual Workshop on Computational Geometry, 1-8.

Miller, E. J. (1997). Towards a 4D GIS: Four-dimensional interpolation utilizing kriging. In Z. Kemp (Ed.), Innovations in GIS: Vol. 4. Innovations in GIS 4. Selected papers from the fourth National Conference on GIS Research UK (GISRUK) (pp. 181-197). London: Taylor \& Francis.

Minkowski, H. (1909). Raum und Zeit. Physikalische Zeitschrift, 10, 104-111.

Minkowski, H. (2012). Space and time. (F. Lewertoff \& V. Petkov, Trans.) V. Petkov (Ed.). Montreal, Quebec: Minkowski Institute Press.

Mitas, L., \& Mitasova, H. (1999). Spatial interpolation. In P. A. Longley, M. F. Goodchild, D. J. Maguire, \& D. W. Rhind (Eds.), Geographical information systems. Principles, techniques, applications, and management (2nd ed., pp. 481-492). New York: Wiley.

Mitasova, H., Mitas, L., Brown, W. M., Gerdes, D. P., Kosinovski, I., \& Baker, T. (1995). Modelling spatially and temporally distributed phenomena: New methods and tools for GRASS GIS. International journal of geographical information systems, 9(4), 433-446.

Montero-Lorenzo, J.-M., Fernández-Avilés, G., Mondéjar-Jiménez, J., \& Vargas-Vargas, M. (2013). A spatio-temporal geostatistical approach to predicting pollution levels: The case of mono-nitrogen oxides in Madrid. Computers, Environment and Urban Systems, 37, 95-106. Retrieved from http://www.sciencedirect.com/science/article/pii/S0198971512000580

Oliver, M. A., \& Webster, R. (1990). Kriging: A method of interpolation for geographical information systems. International journal of geographical information systems, 4(3), 313-332.

Pebesma, E. J. (2004). Multivariable geostatistics in S: the gstat package. Computers \& Geosciences, 30, 683-691.

Pebesma, E. J. (2012). Spacetime: Spatio-Temporal Data in R. Journal of statistical software, 51(7), 130. 
Samet, H. (1995). Spatial Data Structures. In W. Kim (Ed.), Modern Database Systems: The Object Model, Interoperability, and Beyond (pp. 361-385). New York, USA: ACM Press/AddisonWesley Publishing Co.

Sibson, R. (1981). A brief description of natural neighbour interpolation. In V. Barnett (Ed.), Interpreting Multivariate Data (pp. 21-36). Chichester: John Wiley and Sons.

Škala, V. (2016). A practical use of radial basis functions interpolation and approximation. REVISTA INVESTIGACIÓN OPERACIONAL, 37(2), 137-145.

Srinivasan, B. V., Duraiswami, R., \& Murtugudde, R. (2010). Efficient kriging for real-time spatiotemporal interpolation. In American Meteorological Society (Ed.), 20th Conference on Probability and Statistics in the Atmospheric Sciences (P. 228, pp. 1-8).

Stein, M. L. (2013). Interpolation of spatial data: Some theory for kriging. [Place of publication not identified]: Springer.

Susanto, F., Souza, P. de, \& He, J. (2016). Spatiotemporal Interpolation for Environmental Modelling. Sensors (Basel, Switzerland), 16(8), 1-20.

Tomczak, M. (1998). Tomczak, M., 1998. Spatial interpolation and its uncertainty using automated anisotropic inverse distance weighting (IDW)-cross-validation/jackknife approach. Journal of Geographic Information and Decision Analysis, 2(2), 18-30.

Venables, W. N., \& Ripley, B. D. (2002). Modern Applied Statistics with S-Plus. New York: SpringerVerlag.

Wise, S. (2011). Assessing the spatial characteristics of DEM interpolation error through crossvalidation. Computers \& Geosciences, 37(8), 978-991.

Wright, G. B. (2003). Radial Basis Function Interpolation. Numerical and Analytical Development (A thesis submitted to the Faculty of the Graduate School of the University of Colorado in partial fulfillment of the requirements for the degree of Doctor of Philosophy). University of Colorado Boulder, Boulder, Colorado, USA. Retrieved from http://math.boisestate.edu/ wright/research/GradyWrightThesis.pdf 